\title{
The behavioural variant frontotemporal dementia phenocopy syndrome is a distinct entity - evidence from a longitudinal study
}

\author{
E. Devenney ${ }^{1,2^{*}}$, T. Swinn ${ }^{3}$, E. Mioshi ${ }^{4}$, M. Hornberger ${ }^{4}$, K. E. Dawson ${ }^{5}$, S. Mead ${ }^{6}$, J. B. Rowe ${ }^{5}$ and J. R. Hodges ${ }^{1,2}$
}

\begin{abstract}
Background: This study aimed to i) examine the frequency of C9orf72 expansions in a cohort of patients with the behavioural variant frontotemporal dementia (bvFTD) phenocopy syndrome, ii) observe outcomes in a group of phenocopy syndrome with very long term follow-up and iii) compare progression in a cohort of patients with the phenocopy syndrome to a cohort of patients with probable bvFTD.

Methods: Blood was obtained from 16 phenocopy cases. All met criteria for possible bvFTD and were labeled as phenocopy cases if they showed no functional decline, normal cognitive performance on the Addenbrooke's Cognitive Examination-Revised (ACE-R) and a lack of atrophy on brain imaging, over at least 3 years of follow-up. In addition, we obtained very long term follow-up data in 6 cases. A mixed model analysis approach determined the pattern of change in cognition and behaviour over time in phenocopy cases compared to 27 probable bvFTD cases.

Results: All 16 patients were screened for the C9orf72 expansion that was present in only one (6.25\%). Of the 6 cases available for very long-term follow-up (13 - 21 years) none showed progression to frank dementia. Moreover, there was a decrease in the caregiver ratings of behavioural symptoms over time. Phenocopy cases showed significantly slower rates of progression compared to probable bvFTD patients $(p<0.006)$.

Conclusion: The vast majority of patients with the bvFTD phenocopy syndrome remain stable over many years. An occasional patient can harbor the C9orf72 expansion. The aetiology of the remaining cases remains unknown but it appears very unlikely to reflect a neurodegenerative syndrome due to lack of clinical progression or atrophy on imaging.
\end{abstract}

Keywords: Frontotemporal dementia, Phenocopy syndrome, Prognosis, Genetics, Cognition, Behaviour

\section{Background}

The classical features of behavioural variant Frontotemporal Dementia (bvFTD) syndrome are well established. The current consensus criteria incorporates cognitive, behavioural, neuroimaging, genetic and pathological parameters, to provide a framework to make accurate diagnoses by ranking the level of diagnostic certainty as possible, probable and definite [1]. Although the accuracy of these criteria has been pathologically validated, controversy still exists regarding the aetiology, progression and prognosis of possible bvFTD [2].

\footnotetext{
* Correspondence: emma.devenney@sydney.edu.au

${ }^{1}$ Brain and Mind Centre, University of Sydney, Sydney, NSW 2050, Australia

${ }^{2}$ ARC Centre of Excellence in Cognition and its Disorders, Sydney, Australia Full list of author information is available at the end of the article
}

A recent study which followed FTD patients over a five year period found that a number of possible bvFTD patients remain in this category for many years and appear not to progress on cognitive and behavioural measures [3]. A number of these patients are classified as 'phenocopy syndrome' cases [4-7]. Patients harboring the C9orf72 expansion may also satisfy criteria for possible, but not probable, bvFTD at first presentation and may be atypical with pervasive psychotic features [3]. Moreover, cases who have been labeled as the 'phenocopy syndrome' have also been reported to carry the C9orf72 expansion [8, 9]. The question remains, just how many of the phenocopy cases have the expansion?

The present study sought to address this issue by exploring the outcomes in a large and unique cohort of phenocopy

(c) The Author(s). 2018 Open Access This article is distributed under the terms of the Creative Commons Attribution 4.0 International License (http://creativecommons.org/licenses/by/4.0/), which permits unrestricted use, distribution, and 
patients that have been followed over many years and screened for the C9orf 72 expansion. A mixed model analysis was employed to determine the rate of change in global cognition and behaviour over time in these phenocopy cases compared to a group of patients with probable bvFTD.

\section{Methods}

\section{Patients}

Patients were assessed at the specialist early-onset dementia clinic at Addenbrooke's Hospital Cambridge between 1993 and 2007. Patients who satisfied criteria for possible bvFTD only, and were seen on at least two occasions; with initial and follow-up evaluation at least 3 years apart, and in whom blood had been obtained for gene screening, were included in the study. Patients were excluded from the study if they progressed to probable bvFTD over the study period. Exclusion criteria also included a current or past medical history of a psychiatric condition, traumatic brain injury, drug or alcohol abuse and cerebrovascular disease. Of note patients who experienced delusions or hallucinations were included in the study.

Of the 16 cases, three were still under regular review in the clinic in 2014. We attempted to contact the remainder and we able to reassess three additional cases. Thus very long term follow-up (ranging from 13 to 21 years) was available in 6 cases.

A group of probable bvFTD patients were included in the study to serve as a comparison group for the mixed model analysis, to determine differences in progression rates. These patients $(n=27)$ were assessed at FRONTIER, a frontotemporal dementia specialist research clinic and met probable diagnostic criteria for bvFTD. They were matched for age, sex and education to the phenocopy cases. Patients with probable bvFTD who were subsequently found to carry the C9orf72 expansion were not included in this group. None of these patients carried a GRN or MAPT mutation. The results below relate to the phenocopy cases only unless otherwise stated.

Patients were classified according to the current international diagnostic criteria [1]. Patients were classified as possible bvFTD when they met three of the six core behavioural features of bvFTD, but had normal brain imaging and an absence of typical genetic or pathological findings. Probable bvFTD, criteria was met when patients firstly satisfied possible criteria with additional evidence of functional decline, and frontal or temporal abnormalities on MRI or Fludeoxyglucose (18F)-Positron emission tomography (FDG-PET) [1]. In this study MRI scans were performed in all cases and a validated visual rating scale, assessed atrophy of the orbitofrontal cortex, anterior temporal poles and insular cortex, according to previously published data $[10,11]$. Atrophy was rated on a Likert scale by a blinded rater after appropriate training on an independent data set. Intra-class correlation coefficient to assess inter-rater reliability was very high (Cronbach's alpha $=.9)$.

\section{Clinical assessment}

A comprehensive clinical assessment was conducted with the patient and behavioural symptoms were explored with the carer using the CBI (Cambridge Behavioural Inventory), [12]; a higher score indicates greater impairment (maximum score - 316). Global cognitive function was measured using the Addenbrooke's Cognitive Examination-Revised (ACE-R) [13]; a normal score $>88 / 100$.

\section{Genetic screening}

Blood samples were screened at the Medical Research Council Prion Unit, London, or at NeuRA, Sydney, for the C9orf72 expansion based on the repeatprimed polymerase chain reaction technique as previously described by Renton [14]. Genomic DNA was extracted from blood according to standard procedures. Samples were scored as expansion-positive if they harbored $>30$ repeats. C9orf72 hexanucleotide repeat non-expansion alleles were detected by polymerase chain reaction amplification and capillary electrophoresis.

\section{Statistical analysis}

Data were analyzed using SPSS 22.0 statistical package. Normal distribution was determined by means of Kolmogorov-Smirnoff tests. Parametric variables were compared across groups via independent t-tests and analysis of variance (ANOVA). Non-parametric data were analyzed using Mann-Whitney and Kruskal-Wallis tests, and Chi-Square tests compared categorical data. Linear mixed effect models examined change in performance over time [15]. Such measures are useful in these circumstances as they take into account the variability in follow-up time within the phenocopy and probable bvFTD groups, and the significant difference in follow-up between the two groups.

\section{Results \\ Patients}

Between 1993 and 2007 a total of 89 patients with possible bvFTD were assessed and followed for at least 3 years in the specialist clinic. Of these 89 , a diagnosis of probable bvFTD became apparent on follow up in 63 (Fig. 1). The remaining 26 were given a label of phenocopy syndrome on the basis of a lack of progression with relative preservation of activities of daily living, maintained performance on the ACE- $R$ and a normal MRI as assessed by a validated visual rating scale. 


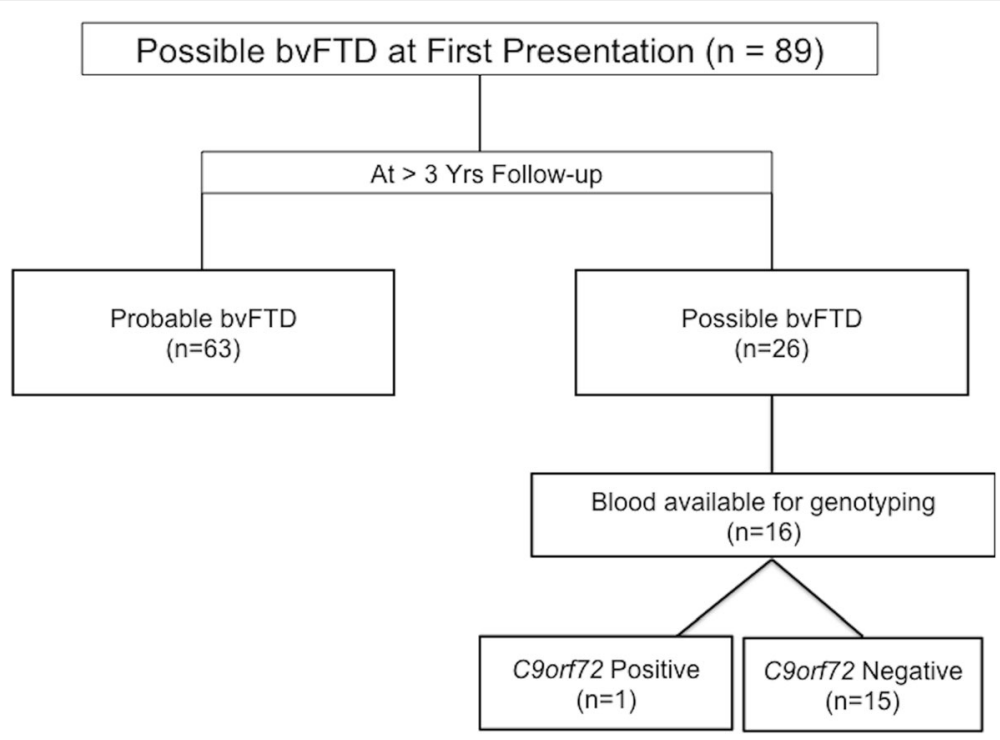

Fig. 1 Longitudinal changes in diagnosis and genetic findings. Flowchart demonstrating the number of patients from the Cambridge cohort at presentation with possible bvFTD, according to diagnostic criteria for bvFTD, and the change in diagnosis and subsequent genetic findings over the follow-up period

Within the comparison bvFTD group, four patients have now come to autopsy and each of these patients showed FTLD pathology including TDP-43 in one, TAU in another and FUS in another.

Blood sampling was obtained in all cases attending the clinic in 2007. In the phenocopy cohort of 26 cases, blood was available in 16 for genotyping.

\section{Genetic testing}

The C9orf72 expansion was present in one of 16 patients who had blood obtained for genotyping, representing 6.25\% of the cohort. This patient was male and in his 60's when he presented with a two-year history of behavioural change. At presentation his score on the CBI of 152 was very high and on the ACE-R his score of 81 was just below the cutoff of 88. A MRI scan was normal. When last seen in 2006 scores, had improved with a CBI score of 136 and an ACE$\mathrm{R}$ score of 89. An FDG-PET scan showed no areas of significant brain hypometabolism. He was then lost to follow up in 2008 (11 years after onset) and died of an unrelated condition in 2010. Unfortunately post mortem brain examination was not performed.

\section{Cognitive and behavioural measures at baseline and follow-up}

The 16 phenocopy patients with available genotyping comprised 15 men with a mean age of 55.7 (range 47 to 69 years). Twelve of the 16 were under 65 . The mean follow-up time was 8 years. Table 1 demonstrates the baseline demographic information for these 16 cases and the comparison group of probable bvFTD patients and includes the mean ACE-R and the CBI scores on first assessment and length of follow-up for the phenocopy and the probable bvFTD group.

At presentation 10 of the 16 phenocopy patients scored above 88 on the ACE-R, and none of the remainder scored below 80/100. At last follow-up six of these patients still scored within the normal range. The profile of behavioural symptoms at presentation and last followup was typical of bvFTD with high endorsements for motivation (apathy), stereotyped and abnormal behaviours, changed appetite and eating and mood.

At presentation there was a significant difference in the ACE-R scores between the phenocopy cases and the probable bvFTD group $(p=0.001)$; the mean ACE-R score in the phenocopy group was $89 / 100$, whereas the mean score for the probable bvFTD group was 73/100.

Table 1 Phenocopy cases - demographic details

\begin{tabular}{llll}
\hline Demographics at Presentation & $\begin{array}{l}\text { Phenocopy } \\
\text { bvFTD } \\
(n=16)\end{array}$ & $\begin{array}{l}\text { Probable } \\
\text { bvFTD } \\
(n=27)\end{array}$ & $P$ value \\
\hline Age at Onset, yrs & $55.7 \pm 6.3$ & $59.7 \pm 8.1$ & 0.1 \\
Sex (M:F) & $15: 1$ & $22: 5$ & 0.4 \\
Disease Duration, yrs. & $3.9 \pm 2.3$ & $3.8 \pm 2.4$ & 0.1 \\
Education, yrs. & $11.4 \pm 2.1$ & $12.4 \pm 3.4$ & 0.3 \\
ACE-R & $89.2 \pm 6.4$ & $72.8 \pm 14.6$ & 0.001 \\
CBI & $91.3 \pm 59$ & $72.5 \pm 21.5$ & 0.3 \\
Follow-up, yrs. & $7.3 \pm 4.2$ & $3.2 \pm 1.3$ & 0.002 \\
\hline
\end{tabular}

Demographic information (Mean \pm standard deviation scores) for the phenocopy cases with blood available for C9orf72 expansion testing, and probable bvFTD cases. bvFTD behavioural variant frontotemporal dementia, ACE-R Addenbrooke's Cognitive Examination-Revised, CBI Cambridge Behavioural Inventory 
In contrast both groups had equivalently high scores on the CBI $(p=0.3)$.

The phenocopy group was then compared to a group of probable bvFTD cases using a mixed model analysis that took into account the variability of follow-up within and between the two groups. On a measure of global cognitive function, the ACE- $R$, the groups combined showed significant deterioration over time $(p<0.001)$ with a significant interaction between disease group and time $(p=0.006)$ indicating a faster rate of decline in probable bvFTD cases compared to phenocopy cases (Fig. 2). On a measure of behaviour, the CBI, the group as a whole showed significant deterioration over time $(p<0.001)$, however while the interaction between disease group and time was not significant there was a statistical trend $(p<0.06)$ suggesting a faster deterioration in behaviour in the probable bvFTD group compared to the phenocopy group.

The mean ACE-R and CBI scores with 95\% confidence intervals, calculated according to the mixed model statistic, for standard times intervals, are demonstrated for the ACE- $\mathrm{R}$ and CBI in Table 2. Table 2 also shows the mean ACE-R and CBI scores at last follow-up for the phenocopy and probable bvFTD group; these are for illustrative purposes only as the follow-up times were variable within and between the groups and therefore statistical analysis based on these measures is not appropriate.

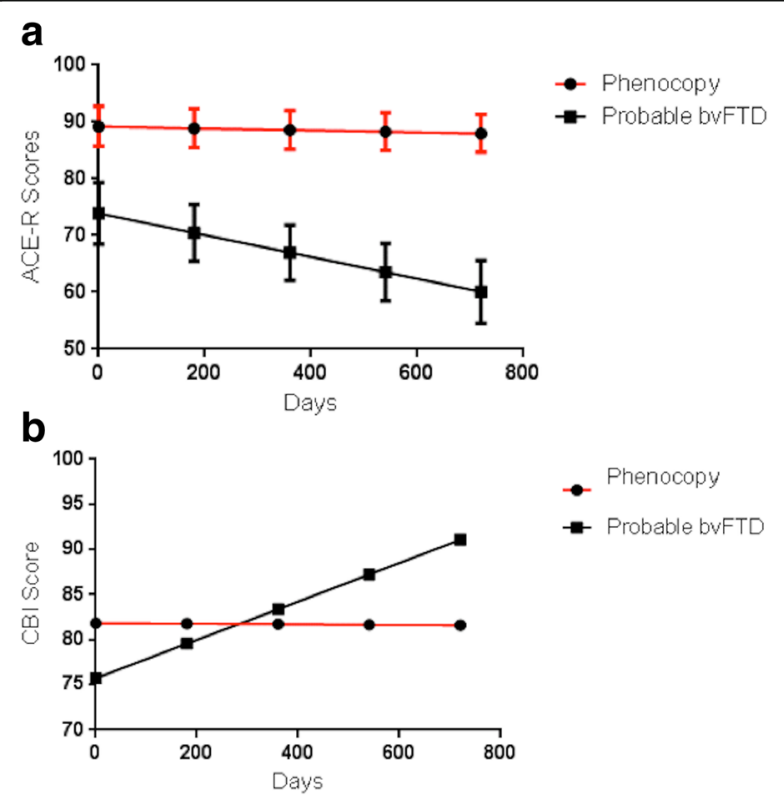

Fig. 2 Longitudinal changes in ACE-R and CBI - phenocopy and bvFTD cases. a demonstrates estimated marginal means based on the \% change in ACE-R score across time for phenocopy and probable bvFTD cases. Time $(p<0.001)$. Time $\times$ Diagnosis $(p=0.006)$. $\mathbf{b}$ demonstrates estimated marginal means based on the change in CBI scores across time. Time $(p<0.001)$. Time $x$ Diagnosis $(p=0.06)$
Table 2 Phenocopy and bvFTD cases - Longitudinal changes in ACE-R and CBI

\begin{tabular}{|c|c|c|}
\hline Time & Phenocopy bvFTD $(n=16)$ & Probable bvFTD $(n=27)$ \\
\hline \multicolumn{3}{|c|}{ ACE-R - Scores (mean, 95\% Cl) } \\
\hline Day 1 & $92(84.1-99.9)$ & $72(65.7-78.3)$ \\
\hline Day 180 & $91.6(84.1-99.2)$ & $69.5(63.8-75.2)$ \\
\hline Day 360 & $91.3(84-98.6)$ & $67(61.5-72.5)$ \\
\hline Day 540 & $91(83.8-98.1)$ & $64.5(58.8-70.2)$ \\
\hline Day 720 & $90.6(83.7-97.5)$ & $62(55.7-68.2)$ \\
\hline \multicolumn{3}{|c|}{ CBI Scores (mean, 95\% Cl) } \\
\hline Day 1 & $81.8(62.7-101)$ & $75.7(60.7-90.7)$ \\
\hline Day 180 & $81.8(63.4-100.1)$ & $79.6(66.1-93)$ \\
\hline Day 360 & $81.7(64.1-99.4)$ & $83.4(70.5-96.3)$ \\
\hline Day 540 & $81.7(64.6-98.8)$ & $87.2(73.9-100.6)$ \\
\hline Day 720 & $81.6(65-98.2)$ & $91.1(76.3-105.9)$ \\
\hline \multicolumn{3}{|c|}{ Last Follow-up (mean, SD) } \\
\hline ACE-R & $85.1 \pm 7.1$ & $54.2 \pm 23.6$ \\
\hline $\mathrm{CBI}$ & $77 \pm 47.3$ & $93.9 \pm 33.7$ \\
\hline
\end{tabular}

Follow-up data according to the mixed model analysis with standard time intervals generated by the model, for phenocopy and probable bvFTD cases. Bottom rows show the mean $\mathrm{ACE}-\mathrm{R}$ and $\mathrm{CBI}$ scores at last follow-up. $\mathrm{Cl}$ confidence interval, $S D$ standard deviation, bvFTD behavioural variant frontotemporal dementia, $A C E-R$ addenbrooke's cognitive examination-Revised, CBI Cambridge behavioural inventory

\section{MRI at baseline and follow-up}

Grey matter density was judged as normal (0) or within normal range (1) in the orbitofrontal cortex, anterior temporal poles and insular cortex in each of the phenocopy cases at baseline and at follow-up.

\section{Long-term clinical follow-up}

In 2013 we attempted to contact the caregivers of the 15 living cases by post and to arrange a telephone interview. Three were still attending the clinic at regular intervals and we were successful in another three (total six), with lengths of follow-up ranging from 13 to 21 years from first visit to the clinic. All were living at home; 3 remained in the same relationship as at their presentation, and there had been no cases with progression to frank dementia.

\section{Discussion}

This study provides evidence for the validity of the bvFTD phenocopy syndrome. Only one of 16 phenocopy cases (6.25\%) had the C9orf72 expansion and it is interesting to note that this is the only patient in the cohort who is known to have died. This study had the benefit of very long-term follow-up information, between 13 and 21 years, in 6 cases. There was no evidence of progression to frank dementia in any of the phenocopy cases over many years of follow-up.

The underlying aetiology of the phenocopy syndrome is unknown. On a clinical level, these patients present 
with cognitive and behavioural changes, that are identical to the deficits seen in probable bvFTD cases, yet do not show significant brain atrophy $[6,16]$. Furthermore, a previous clinicopathological study found that 2 phenocopy cases did not have FTLD pathology at autopsy [17]. While it is possible that the phenocopy syndrome represents a late onset decompensated developmental disorder in the Asperger-Autism spectrum, it remains to be proven. In keeping with this hypothesis, such patients, although scoring normally on tests such as the ACE-R and measures of memory, may show mild deficits on tests of inhibitory control and emotion processing [18] as do patients on the Asperger-Autism spectrum $[19,20]$. A recent study comparing phenocopy and probable bvFTD cases showed a high rate of adverse life events, relationship problems and cluster $\mathrm{C}$ personality traits comprising the avoidant, dependent, and obsessive-compulsive personality traits [21]. Putting these findings together it seems highly likely that the phenocopy syndrome is a final common pathway for a complex interaction of a number of personality and psychiatric factors.

Interestingly long-term assessments show that, although some patients continue to exhibit behavioural symptoms, these symptoms are rated as less marked by caregivers over time. This could, of course, simply reflect the fact that family members adjust to and are less troubled by the symptoms. A study of possible bvFTD patients followed over several years showed that a subgroup, many of whom had the C9orf72 expansion, progressed on cognitive and functional measures while others, who lacked the expansion, demonstrated no change and conformed, therefore, to the phenocopy syndrome [3]. Although the former work did not have the benefit of such long-term followup, it mirrors the results from this study, which showed that the phenocopy cases did not progress on the $\mathrm{CBI}$, and together these findings point towards a nonprogressive non-neurodegenerative aetiology in phenocopy cases. The probable bvFTD patients were also significantly more impaired on the ACE- $\mathrm{R}$ at presentation, and the mixed model analysis revealed a significant deterioration in ACE-R scores over time in the probable bvFTD group compared to the phenocopy group further demonstrating the relative cognitive stability of phenocopy cases.

Studies of FTD have established that the C9orf72 expansion, whilst variable in prevalence around the world, is a common Mendelian genetic cause of familial disease, and is also present in a proportion of sporadic cases [22]. The full clinical spectrum associated with the expansion is not yet clear but it has been shown that such patients have a high rate of psychotic symptoms and that there is considerable variability in the rate of progression. While some patients present with a long insidious history of gradual decline others have a more fulminating illness $[8,9]$. Studies have also linked C9orf72 to other clinical phenotypes outside of FTD and MND, including Parkinson's disease, multiple system atrophy (MSA) and Alzheimer's disease (AD), although many lacked neuropathological confirmation [22]. Nonetheless, there does appear to be partial penetrance as C9orf72 carriers may remain asymptomatic into their 80's [23]. We have confirmed that patients with the phenocopy syndrome may also harbor the expansion but in a well-characterized cohort with long term follow up this appears to be the exception. Interestingly, the only C9orf 72 carrier in our phenocopy cohort did not show any abnormalities on MRI or FDG-PET, in keeping with reports from prior studies $[8,9]$. Our work provides data to support the informed genetic counseling of this clinical group. A lack of understanding of the phenocopy syndrome and support for the patients and their families can make recruitment into a research programme difficult. Nonetheless further work is necessary to confirm the proportion of the FTD phenocopy syndrome that has a genetic aetiology and also to confirm the underlying pathology in these cases. Moreover, study of the phenocopy syndrome may help clarify the link between psychiatric illness and frontotemporal dementia. As in this project, cases that have a psychiatric history are usually excluded from studies however this design may need to be reconsidered in the future in light of this apparent link and co-existence of psychiatric and neurodegenerative disorders.

\section{Conclusion}

We propose that the phenocopy syndrome is a valid entity. These patients are almost always male and experience symptom onset between the ages of 45 and 65 . Despite reported behavioural changes, they perform relatively normally on general cognitive tests such as the ACE-R or ACE-III, have preserved basic activities of daily living, lack atrophy on MRI and critically show no decline after 3 years of follow-up. Within the first two to 3 years of evaluation of possible bvFTD cases physicians should exhibit caution in diagnosing the phenocopy syndrome, since the majority will progress to probable disease over time and almost one half will progress within the first 3 years. It should be also stressed that although phenocopy cases may not harbour underlying neurodegenerative pathology, this is not a benign condition and caregiver burden can be high.

\section{Abbreviations}

ACE-R: Addenbrookes cognitive examination - Revised; bvFTD: behavioural variant frontotemporal dementia; CBI: Cambridge behavioural inventory; FDG-PET: Fludeoxyglucose (18F)-Positron emission tomography

\section{Acknowledgements}

We are grateful to the research participants involved with the research studies. Work at University College London Institute of Neurology was supported by the Medical Research Council (UK) and the National Institute of Health Research's Biomedical Research Centre at University College London Hospital. 


\section{Funding}

This work was supported by funding to Forefront, a collaborative research group dedicated to the study of frontotemporal dementia and motor neurone disease, from the National Health and Medical research Council of Australia program grant (\#1037746) and the Australian Research Council Centre of Excellence in Cognition and its Disorders Memory Node (\#CE110001021). The funding body did not have a role in the design of the study and collection, analysis, and interpretation of data and in writing the manuscript.

Dr. Devenney is supported by the Motor Neurone Disease Research Institute Australia. Professor Eneida Mioshi is supported by the Alzheimer's Association (USA) and the Alzheimer's Society (UK). Professor Michael Hornberger is supported by Alzheimer's Research UK, Medical Research Council and the Wellcome Trust. Professor James Rowe is supported by the Wellcome Trust (\#103838) and the National Institute for Health Research Cambridge Biomedical Research Centre.

\section{Availability of data and materials}

All data generated or analysed during this study are included in this published article.

\section{Authors' contributions}

ED contributed to the study design, data analysis, manuscript preparation, writing and review. TS contributed to study design, data collection, and review. EM, MH, SM, JRH contributed to study design, data collection, manuscript preparation and review. KED contributed to study design, data collection and review. JBR contributed to study design, data collection, data analysis, manuscript preparation and review. All authors read and approved the final manuscript

\section{Ethics approval and consent to participate}

Ethical approval was obtained from the Cambridge research ethics committee (\#07/Q0102/3) as part of the NHS National Research Ethics Service, and the South Eastern Sydney local Health District and the University of New South Wales human research ethics committee (\#10/126). Patients, or their legal representatives acting as proxy, provided informed written consent in accordance with the Declaration of Helsinki.

\section{Competing interests}

Professor E Mioshi was previously a member of the editorial board for BMC Neurology. On behalf of all the other authors, the corresponding author states that there is no competing interest.

\section{Publisher's Note}

Springer Nature remains neutral with regard to jurisdictional claims in published maps and institutional affiliations.

\section{Author details}

'Brain and Mind Centre, University of Sydney, Sydney, NSW 2050, Australia. ${ }^{2}$ ARC Centre of Excellence in Cognition and its Disorders, Sydney, Australia. ${ }^{3}$ Medical Research Council Cognition and Brain Sciences Unit, Cambridge, UK. ${ }^{4}$ Faculty of Medicine and Health Sciences, University of East Anglia, Norwich, UK. ${ }^{5}$ Department of Clinical Neurosciences, University of Cambridge, Cambridge, UK. ${ }^{6}$ MRC Prion Unit, Department of Neurodegenerative Disease, UCL Institute of Neurology, Queen Square, London, UK

\section{Received: 3 November 2017 Accepted: 20 April 2018}

\section{Published online: 28 April 2018}

\section{References}

1. Rascovsky K, Hodges JR, Knopman D, Mendez MF, Kramer JH, Neuhaus J, van Swieten JC, Seelaar H, Dopper EG, Onyike CU, et al. Sensitivity of revised diagnostic criteria for the behavioural variant of frontotemporal dementia. Brain. 2011;134(Pt 9):2456-77.

2. Chare L, Hodges JR, Leyton CE, McGinley C, Tan RH, Kril JJ, Halliday GM. New criteria for frontotemporal dementia syndromes: clinical and pathological diagnostic implications. J Neurol Neurosurg Psychiatry. 2014; 85(8):865-70.

3. Devenney E, Bartley L, Hoon C, O'Callaghan C, Kumfor F, Hornberger M, Kwok JB, Halliday GM, Kiernan MC, Piguet O, et al. Progression in
Behavioural variant frontotemporal dementia: a longitudinal study. JAMA Neurol. 2015:72(12):1501-9.

4. Hornberger M, Shelley BP, Kipps CM, Piguet O, Hodges JR. Can progressive and non-progressive behavioural variant frontotemporal dementia be distinguished at presentation? J Neurol Neurosurg Psychiatry. 2009;80(6):591-3.

5. Hornberger M, Piguet O, Kipps C, Hodges JR. Executive function in progressive and nonprogressive behavioural variant frontotemporal dementia. Neurology. 2008;71(19):1481-8.

6. Kipps CM, Hodges JR, Fryer TD, Nestor PJ. Combined magnetic resonance imaging and positron emission tomography brain imaging in behavioural variant frontotemporal degeneration: refining the clinical phenotype. Brain. 2009;132(Pt 9):2566-78.

7. Mioshi E, Hsieh S, Savage S, Hornberger M, Hodges JR. Clinical staging and disease progression in frontotemporal dementia. Neurology. 2010;74(20):1591-7.

8. Khan BK, Yokoyama JS, Takada LT, Sharon JS, Rutherford NJ, Fong JC, Karydas AM, Wu T, Ketelle RS, Baker MC. Atypical, slowly progressive behavioural variant frontotemporal dementia associated with C90RF72 hexanucleotide expansion. J Neurol Neurosurg Psychiatry. 2012;83(4):358-64.

9. Devenney E, Foxe D, Dobson-Stone C, Kwok JB, Kiernan MC, Hodges JR: Clinical heterogeneity of the C9orf72 genetic mutation in frontotemporal dementia.Neurocase 2014(ahead-of-print):1-7.

10. Kipps CM, Davies RR, Mitchell J, Kril JJ, Halliday GM, Hodges JR. Clinical significance of lobar atrophy in frontotemporal dementia: application of an MRI visual rating scale. Dement Geriatr Cogn Disord. 2007;23(5):334-42.

11. Ambikairajah A, Devenney E, Flanagan E, Yew B, Mioshi E, Kiernan MC, Hodges JR, Hornberger M. A visual MRI atrophy rating scale for the amyotrophic lateral sclerosis-frontotemporal dementia continuum. Amyotroph Lateral Scler Frontotemporal Degener. 2014;0:1-9.

12. Wedderburn C, Wear H, Brown J, Mason SJ, Barker RA, Hodges J, WilliamsGray C. The utility of the Cambridge Behavioural inventory in neurodegenerative disease. J Neurol Neurosurg Psychiatry. 2008;79(5):500-3.

13. Mioshi E, Dawson K, Mitchell J, Arnold R, Hodges JR. The Addenbrooke's cognitive examination revised (ACE-R): a brief cognitive test battery for dementia screening. Int J Geriatr Psychiatry. 2006;21(11):1078-85.

14. Renton AE, Majounie E, Waite A, Simón-Sánchez J, Rollinson S, Gibbs JR, Schymick JC, Laaksovirta H, Van Swieten JC, Myllykangas L. A Hexanucleotide repeat expansion in $<\mathrm{i}>\mathrm{C}$ (ORF72 $</ \mathrm{i}>$ is the cause of chromosome 9p21-linked ALS-FTD. Neuron. 2011;72(2):257-68.

15. Laird NM, Ware JH. Random-effects models for longitudinal data. Biometrics. 1982;38(4):963-74.

16. Kipps CM, Hodges JR, Hornberger M. Nonprogressive behavioural frontotemporal dementia: recent developments and clinical implications of the 'bvFTD phenocopy syndrome'. Curr Opin Neurol. 2010;23(6):628-32.

17. Devenney E, Forrest SL, Xuereb J, Kril JJ, Hodges JR. The bvFTD phenocopy syndrome: a clinicopathological report. J Neurol Neurosurg Psychiatry. 2016; 87(10):1155-6.

18. Kumfor F, Irish M, Leyton C, Miller L, Lah S, Devenney E, Hodges JR, Piguet $O$. Tracking the progression of social cognition in neurodegenerative disorders. J Neurol Neurosurg Psychiatry. 2014;85(10):1076-83.

19. Happé $F$, Booth $R$, Charlton R, Hughes C. Executive function deficits in autism spectrum disorders and attention-deficit/hyperactivity disorder: examining profiles across domains and ages. Brain Cogn. 2006;61(1):25-39.

20. Ashwin C, Chapman E, Colle L, Baron-Cohen S. Impaired recognition of negative basic emotions in autism: a test of the amygdala theory. Soc Neurosci. 2006;1(3-4):349-63.

21. Gossink FT, Dols A, Kerssens CJ, Krudop WA, Kerklaan BJ, Scheltens P, Stek ML, Pijnenburg YA. Psychiatric diagnoses underlying the phenocopy syndrome of behavioural variant frontotemporal dementia. J Neurol Neurosurg Psychiatry. 2016:87(1):64-8.

22. Beck J, Poulter M, Hensman D, Rohrer JD, Mahoney CJ, Adamson G, Campbell T, Uphill J, Borg A, Fratta P. Large C9orf72 hexanucleotide repeat expansions are seen in multiple neurodegenerative syndromes and are more frequent than expected in the UK population. Am J Hum Genet. 2013; 92(3):345-53.

23. Galimberti D, Arosio B, Fenoglio C, Serpente M, Cioffi SM, Bonsi R, Rossi P, Abbate C, Mari D, Scarpini E. Incomplete penetrance of the C9ORF72 hexanucleotide repeat expansions: frequency in a cohort of geriatric nondemented subjects. J Alzheimers Dis. 2014;39(1):19-22. 